\title{
Megf10 Is a Receptor for C1Q That Mediates Clearance of Apoptotic Cells by Astrocytes
}

\author{
Tal Iram, ${ }^{1,2,3}$ Zaida Ramirez-Ortiz, ${ }^{1}$ Michael H. Byrne, ${ }^{1}$ Uwanda A. Coleman, ${ }^{1}$ Nathan D. Kingery, ${ }^{1}$ Terry K. Means, ${ }^{1}$ \\ Dan Frenkel, ${ }^{2,3}$ and Joseph El Khoury ${ }^{1,4}$ \\ ${ }^{1}$ Center for Immunology and Inflammatory Diseases and Division of Rheumatology, Allergy, and Immunology, Massachusetts General Hospital and \\ Harvard Medical School, Boston, Massachusetts 02114, ${ }^{2}$ Sagol School of Neuroscience and ${ }^{3}$ Department of Neurobiology, George S. Wise Faculty of Life \\ Sciences, Tel Aviv University, Tel Aviv, Israel 69978, and ${ }^{4}$ Division of Infectious Diseases, Massachusetts General Hospital, Boston, Massachusetts 02114
}

Multiple EGF-like domains 10 (Megf10) is a class F scavenger receptor (SR-F3) expressed on astrocytes and myosatellite cells, and recessive mutations in humans result in early-onset myopathy, areflexia, respiratory distress, and dysphagia (EMARDD). Here we report that Megf10-deficient mice have increased apoptotic cells in the developing cerebellum and have impaired phagocytosis of apoptotic cells by astrocytes ex vivo. We also report that cells transfected with Megf10 gain the ability to phagocytose apoptotic neurons and that Megf10 binds with high affinity to $\mathrm{Clq}$, an eat-me signal for apoptotic cells. In contrast, cells expressing Megf 10 with EMARDD mutations have impaired apoptotic cell clearance and impaired binding to C1q. Our studies reveal that Megf10 is a receptor for C1q and identify a novel role for Megf10 in clearance of apoptotic cells in the mammalian developing brain with potential relevance to EMARDD patients and other CNS disorders.

Key words: apoptosis; astrocytes; C1q; clearance; EMARDD; MEGF10; scavenger receptor, SR-F3

\section{Significance Statement}

Apoptosis is a universal homeostatic process and occurs in many disease conditions. Multiple EGF-like domains 10 (Megf10) is emerging as an essential receptor for synaptic pruning, clearance of neuronal debris, and for muscle differentiation. Here we define a novel Megf10-dependent pathway for apoptotic cell clearance and show that Megf10 is a receptor for C1q, an eat-me signal, that binds phosphatidylserine expressed on the surface of apoptotic cells. Understanding the pathways by which apoptotic cells are cleared in the CNS is relevant to many physiological and pathological conditions of the CNS.

\section{Introduction}

Clearance of apoptotic cells is an important homeostatic process in the CNS. Extensive work has been done on the clearance of apoptotic cells in Caenorhabditis elegans and Drosophila, and the receptors cell death abnormality protein 1 (CED-1) and its ortholog Draper have been implicated in this process (Zhou et al., 2001; Freeman et al., 2003). In the mammalian central nervous system (CNS), the receptors involved in the clearance of apoptotic cells in vivo have not been definitively identified yet. Astrocytes

Received 0ct. 23, 2015; revised Feb. 26, 2016; accepted March 31, 2016.

Author contributions: T.I., T.K.M., D.F., and J.E.K. designed research; T.I., M.H.B., U.A.C., and N.D.K. performed research; Z.R.-0. contributed unpublished reagents/analytic tools; T.I., Z.R.-O., N.D.K., T.K.M., D.F., and J.E.K. analyzed data; T.I., D.F., and J.E.K. wrote the paper.

This work is supported by National Institutes of Health Grants U24 Al082660 (J.E.K.) and R01-Al084884 (T.K.M.), Alzheimer's Association Grant NIRG-11-205535, and a grant from the Ministry of Science, Technology, and Space (D.F.). During this research, T.I. received a student fellowship from the Israeli National Network of Excellence in Neuroscience funded by Teva Pharmaceutical Industries and a student fellowship from the Clore Israel Foundation. We thank Prof. Zvi Fishelson (Tel Aviv University, Tel Aviv, Israel) for critical reading of the manuscript.

Correspondence should be addressed to Joseph El Khoury, Division of Infectious Diseases, Massachusetts General Hospital, 55 Fruit Street, Boston, MA 02114. E-mail: jelkhoury@mgh.harvard.edu.

DOI:10.1523/JNEUROSCI.3850-15.2016

Copyright $\odot 2016$ the authors $\quad 0270-6474 / 16 / 365185-08 \$ 15.00 / 0$ can promote debris clearance and tissue repair in several brain pathologies (Wyss-Coray et al., 2003; Faulkner et al., 2004; Iram and Frenkel, 2012). Astrocytes are enriched with orthologs of the CED family phagocytic-pathway genes, especially with the class $\mathrm{F}$ scavenger receptor SR-F3 also known as multiple EGF-like domains 10 (Megf10), a mammalian ortholog of CED-1 and Draper (Cahoy et al., 2008), that mediates engulfment of synapses by astrocytes in the developing and adult CNS (Chung et al., 2013; Prabhudas et al., 2014). In vitro studies also show that Megf10 expressed by glial precursors in spinal cord dorsal root ganglia can bind apoptotic neurons and mediate their engulfment in culture (Wu et al., 2009; Scheib et al., 2012). The role of Megf10 in apoptotic cell engulfment in vivo in the brain or cerebellum (CB) is not known.

In this study, we investigated the role of Megf10 in the clearance of apoptotic cells in vivo using $M e g f 10^{-1-}$ mice and found that even partial deficiency in Megf10 impairs the ability of astrocytes to clear apoptotic cells in the developing CB.

\section{Materials and Methods}

Animals. All mice were maintained in specific pathogen-free conditions at the Massachusetts General Hospital animal care facility under a pro- 
tocol approved by the Institutional Animal Care and Use Committee. Wild-type (WT) C57BL/6 mice were purchased from National Institutes of Health (NIH). Megf10 $10^{-1-}$ mice were made at the Mutant Mouse Regional Resource Center on a C57BL/6NTac background and bred inhouse with C57BL/ 6 from the NIH for at least nine generations. Briefly, exons 1-24 of mouse Megf10 (mMefg10) was replaced by homologous recombination with an expression selection cassette as detailed by the Knockout Mouse Project (KOMP, University of California Davis, Davis, CA; https://www.komp.org/geneinfo.php?geneid=68051). The colony was maintained as Megf $10^{+/-}$. Megf $10^{-/-}, \mathrm{Megf} 10^{+/-}$, and WT littermate animals used in the experiments were generated by breeding Megf $10^{+/-}$together. All experiments were done using male mice.

Expression vectors generation: subcloning of human full-length MEGF10 into $p C D N A 3.1+$ for expression in mammalian cells. A full-length human Megf10 (hMegf10) cDNA clone (catalog \#TC104847; Origene Technologies) was subcloned into the pCDNA3.1+ vector (catalog \#V790-20; Invitrogen Life Technologies). Final confirmatory sequencing was done before the plasmid was used for expression in mammalian cells. For the binding experiments, the extracellular domain of hMegf10 (ex-hMegf10) encoding amino acids 1-857 was generated by PCR from the hMegf10 cDNA clone and then subcloned into the p3XFlag-CMV-14 vector (catalog \#E7908; Sigma-Aldrich) to add a C-terminal $6 \times$ His $-3 \times$ Flag fusion tag and allow for mammalian cell expression. To generate red fluorescent protein (RFP)-labeled Megf10, full-length mMegf10 c-DNA (catalog \#MMM1013-9498084; Thermo Fisher Scientific) was subcloned into the pCMV6-AC-RFP. A control vector was constructed to express RFP only without the Megf10 fusion partner. All cDNA clones used in this study were sequenced in full to confirm their integrity.

$m$ Megf10-GFP transfection and apoptotic cell engulfment experiments. For engulfment experiments, human embryonic kidney cells (HEK293T) were transfected with either control RFP or mMegf10-RFP vectors using Lipofectamine 2000 (Invitrogen Life Technologies) following the instructions of the manufacturer. Experiments were conducted $48 \mathrm{~h}$ after transfection.

Apoptosis of mouse embryonic fibroblasts (MEFs) and catecholaminergic neuron cell line (CAD) was induced by UV radiation as described previously (Ramirez-Ortiz et al., 2013). For engulfment experiments, apoptotic MEFs or CAD neurons were labeled with $2.86 \mu \mathrm{M}$ DAPI and incubated for $30 \mathrm{~min}$ at $37^{\circ} \mathrm{C}$. Apoptosis induction was validated in an aliquot of these cells by staining with annexin $\mathrm{V}$ and propidium iodide (both from R \& D Systems) and measured by flow cytometry. Apoptotic cells were washed, suspended in the media of engulfing cells, and put on engulfing cells at a ratio of $1: 1$ for $4 \mathrm{~h}$ at $37^{\circ} \mathrm{C}$. Measurement of engulfment was done by imaging the eight-chamber slide using a Nikon Eclipse ME600 fluorescent microscope equipped with a high-resolution DXM1200C Nikon digital camera. Engulfment was determined for each well by the percentage of at least 100 red cells (either RFP or Megf10RFP) containing at least one apoptotic cell.

C1q labeling. Human C1q (Complement Technologies) was conjugated to Alexa-Fluor 647 using amine-reactive probes (Invitrogen) following the instructions of the manufacturer. Briefly, Alexa-Fluor 647 in $0.1 \mathrm{~m}$ sodium bicarbonate, $\mathrm{pH} 8.3$, was mixed with $\mathrm{C} 1 \mathrm{q}$ for $1 \mathrm{~h}$ at room temperature. Unbound Alexa-Fluor 647 was removed using a size separation column.

hMegf10-GFP transfection and C1q binding experiments. Full-length hMegf10 in pCMV6-AC-GFP expression vector (catalog \#RG217883; Origene Technologies) was used for site-directed mutagenesis experiments. Mutations C326R and C774R as described previously (Logan et al., 2011; Boyden et al., 2012) were introduced by site-directed mutagenesis (GenScript Mutagenesis Services). HEK-293T cells were transfected with GFP, full-length hMegf10-GFP, or hMegf10 with EMARDD mutations $48 \mathrm{~h}$ before $1 \mathrm{~h}$ incubation with the indicated concentrations of labeled C1q. Cells were washed, and the percentage of C1q uptake was analyzed by flow cytometry. For imaging, HEK cells were transfected with untagged hMegf10 and incubated with $15 \mu \mathrm{g} / \mathrm{ml}$ labeled C1q overnight with and without apoptotic MEFs labeled with red nuclear mask in a 1:1 ratio (Invitrogen) and then fixed and stained with rabbit antiMegf10 antibody (1:200; Atlas Antibodies) and goat anti-rabbit Alexa-
Fluor 488 (Invitrogen) as secondary antibody. Slides were imaged by confocal microscopy (LSM 780; Zeiss).

Primary astrocyte culture and engulfment experiments. Brains from 3-month-old C57B6, Megf $10^{+/-}$, and Megf10 $0^{-/-}$were harvested and dissociated using a papain neural tissue dissociation kit (Miltenyi) following the instructions of the manufacturer. Myelin was separated by spinning the cells in $30 \%$ Percoll gradient for $40 \mathrm{~min}$ at $850 \times \mathrm{g}$ following a wash step and red blood cells lysis. Cells were placed on poly-D-lysinecovered plates and grown with astrocyte medium (ScienCell) for 2-3 weeks. For engulfment experiments by primary adult astrocytes, apoptotic cells were labeled for 30 min with YoPRO-A488 (Invitrogen Life Technologies), washed, and incubated with astrocytes for 4 hours at $37^{\circ} \mathrm{C}$. After an extensive wash, astrocytes were detached in a non-enzymatic manner, spun and resuspended in cell permeabilization/fixation buffer for $30 \mathrm{~min}$ on ice (R \& D Systems), washed in PBS, and stained with mouse anti-GFAP-Alexa-Fluor 647 antibody (Invitrogen Life Technologies) in permeabilization buffer (R \& D Systems) containing 1\% FCS and 1:100 FcR block (BioLegend) for $30 \mathrm{~min}$. Cells were washed and analyzed in an Accuri C6 flow cytometer.

Soluble ex-hMegf10 purification and Western blot. Supernatant collected from HEK-293 expressing the soluble ex-hMegf10 (6×His$3 \times$ Flag tagged) was passed through a nickel purification column (Thermo Fisher Scientific) and eluted in $0.25 \mathrm{M}$ imidazole in $1 \mathrm{ml}$ fractions. Fractions containing Megf10 were identified by Western blot using rabbit anti-hMegf10 (Sigma-Aldrich), followed by anti-rabbit HRPconjugated antibody (1:10,000; Sigma-Aldrich) for $1 \mathrm{~h}$ at room temperature and detected by chemiluminescence (GE Healthcare). For anti-Flag immunoblotting, membrane was blocked $1 \mathrm{~h}$ in $0.5 \%$ BSA in TBST, washed, and incubated with anti-Flag HRP-conjugated antibody (1: 10,000; Sigma-Aldrich) for $1 \mathrm{~h}$ in $0.5 \%$ BSA in TBST. Binding was detected by chemiluminescent detection (GE Healthcare). The fractions that had Megf10-positive bands were pooled and concentrated using Amicon Ultra $30 \mathrm{kDa}$ molecular weight cutoff (Millipore).

Dot blot and binding assays. Membrane lipid strips (Echelon Bioscience) spotted with 100 pmol of 10 membrane lipids were blocked for $1 \mathrm{~h}$ with $5 \%$ BSA in TBST ( $50 \mathrm{~mm}$ Tris, $0.5 \mathrm{M} \mathrm{NaCl}$, and $0.05 \%$ Tween 20 , pH 7.4). After blocking, membranes are incubated with $15 \mu \mathrm{g}$ of purified ex-hMegf10 protein for $1 \mathrm{~h}$ in $0.5 \%$ BSA in TBST, washed, and incubated with anti-Flag (1:10,000; Sigma-Aldrich) for $1 \mathrm{~h}$ in $0.5 \%$ BSA in TBST. Binding was detected by chemiluminescent detection (GE Healthcare). For C1q binding, $1 \mu \mathrm{g}$ of recombinant protein (3C1740; Sigma-Aldrich) was blotted on a PVDF membrane (Invitrogen Life Technologies) and then followed the same protocol as the membrane lipid strips above.

Surface plasmon resonance. Binding experiments were performed by Precision Antibody on a Biacore 3000 at $25^{\circ} \mathrm{C}$. Seven hundred relative units of His-Ex-hMegf10 was directly immobilized on flow cell 2 of CM5 chip by amine coupling [1-ethyl-3-(3-dimethylaminopropyl)carbodiimide/ $N$-hydroxysulfosuccinimide], and unoccupied sites were blocked with $1 \mathrm{~m}$ ethanol amine. Flow cell 1 used as a blank reference surface. Phosphatidylserine (PS; catalog \#L3116; Echelon Bioscience) was flowed over the chip at a single analyte concentration as indicated. For C1q (catalog \#A099; Complement Technologies), a full kinetic analysis was performed at analyte concentration $25 \mathrm{nM}$, followed by serial dilution to $12.5,6.25$, and $0 \mathrm{~nm}$. Binding of analyte to the ligand was monitored in real time to obtain on $\left(k_{a}\right)$ and off $\left(k_{d}\right)$ rates. The equilibrium constant $\left(K_{D}\right)$ was calculated from the observed $k_{a}$ and $k_{d} \cdot \chi^{2}$ analysis was performed between the actual sensorgram (colored line) and the sensorgram generated from the BIAnalysis software (black line) to determine the accuracy of the analysis. A $\chi^{2}$ value within $1-2$ is considered significant (accurate) and $<1$ is highly significant (highly accurate).

Real-time quantitative PCR. Total RNA was extracted using the RNeasy micro-kit according to the protocol of the manufacturer (QIAGEN), and each sample was reverse transcribed using SuperScript III polymerase (Invitrogen Life Technologies). RT-PCR reaction was done with Syber Green reagent (Roche) in a LightCycler480II machine (Roche).

Terminal deoxynucleotidyl transferase-mediated biotinylated UTP nick end labeling assay. Frozen sections of postnatal day 7 (P7) WT, $\mathrm{Megf} 10^{+/-}$, and $\mathrm{Meg} f 10^{-1-}$ were allowed to warm to room temperature 
A

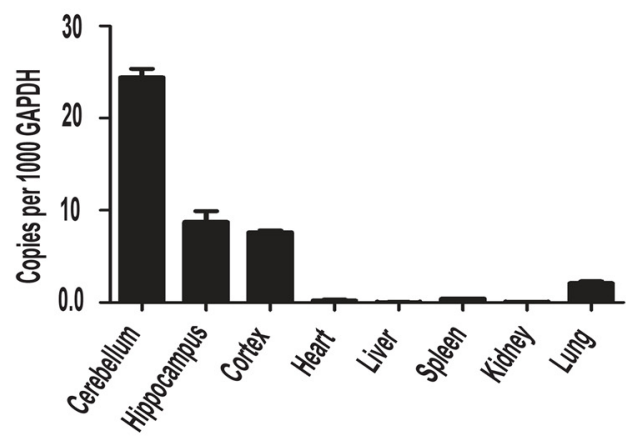

D

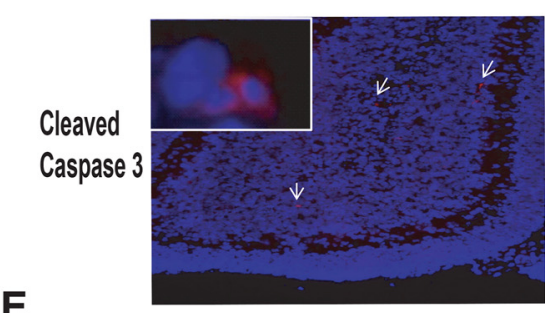

E

TUNEL

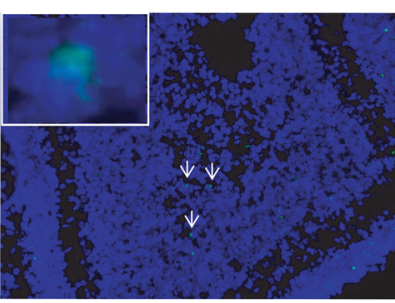

$\mathbf{F}$

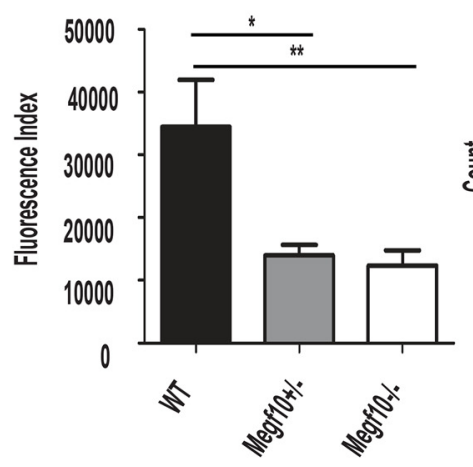

H

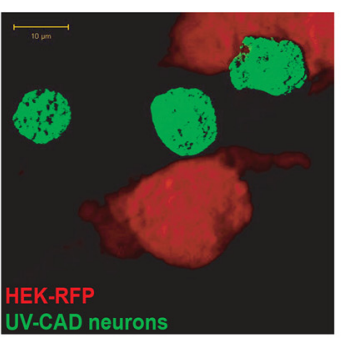

B

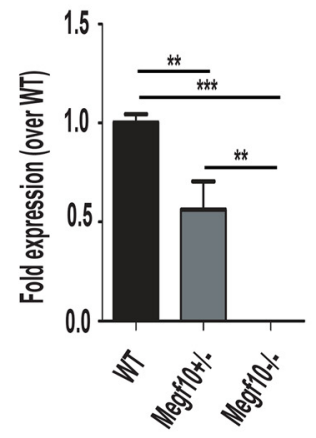

C

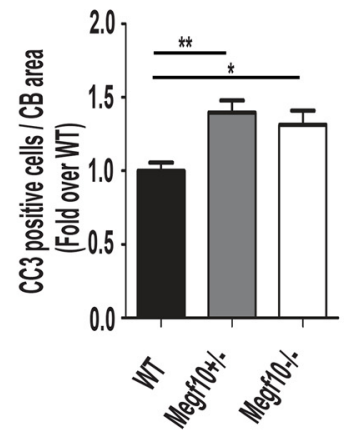

Megf10t+-


G

Megf10-/-
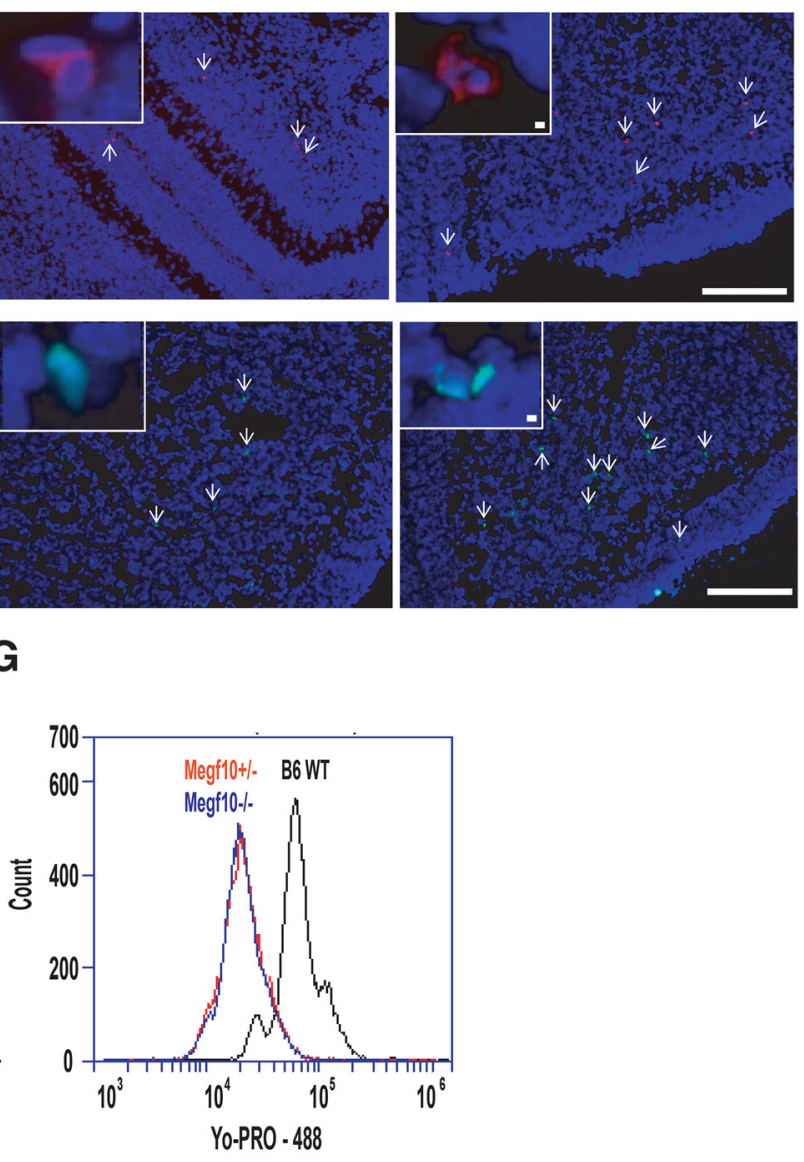

$\mathbf{J}$

$\mathbf{K}$
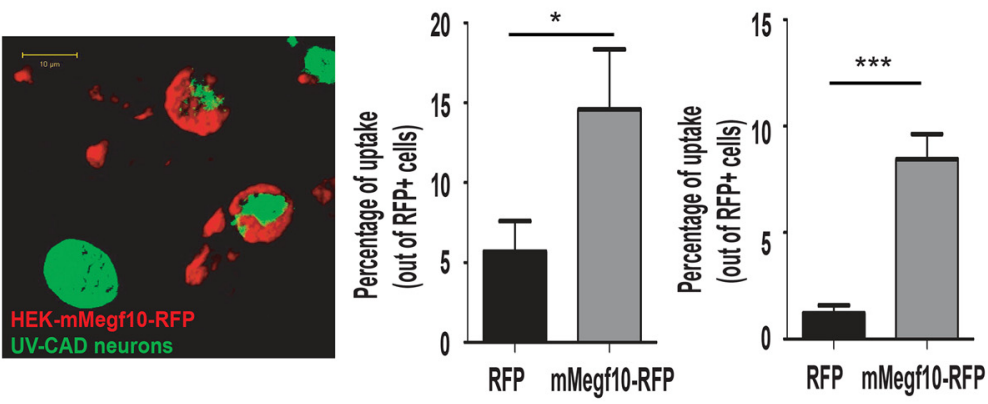

Figure 1. Megf10 is necessary for apoptotic cell uptake by astrocytes, and its deficiency results in accumulation of apoptotic cells in the developing CB. $A$, Megf10 mRNA expression in different organs from C57B6 WT male mice as measured by RT-PCR $(n=3)$. B, Megf10 expression in the CB of P7 WT, Megf10 ${ }^{+-}$, and Megf10 ${ }^{-1-}$ mice $(n=3-5 ; p<0.01)$. C, Quantification of anti-CC3




and fixed in $4 \%$ paraformaldehyde for $1 \mathrm{~h}$. Terminal deoxynucleotidyl transferase-mediated biotinylated UTP nick end labeling (TUNEL) assay (Roche) for detection of apoptotic cells was performed following the instructions of the manufacturer. Positive control was incubated with DNase 1 (3-3000 U/ml in $50 \mu \mathrm{M}$ Tris- $\mathrm{HCl}, \mathrm{pH}$ 7.5, $10 \mathrm{~mm} \mathrm{MgCl}_{2}$, and 1 $\mathrm{mg} / \mathrm{ml} \mathrm{BSA}$ ) for $10 \mathrm{~min}$ at room temperature before the TUNEL incubation. Finally, DNA was stained with DAPI for $2 \mathrm{~min}$ at room temperature. Slides were mounted using Vectamount (Vector Laboratories) mounting media and visualized using a Nikon Eclipse ME600 fluorescent microscope equipped with a high-resolution DXM1200C Nikon digital camera. Data were analyzed using NIS-Elements software (Nikon).

Immunofluorescence. P7 brains were harvested, sectioned, and stained with rabbit anti-cleaved caspase 3 (CC3) overnight (1:200 in blocking buffer; Cell Signaling Technology) and then with secondary antibody Alexa-Fluor 555 donkey anti-rabbit (1:500 in blocking buffer; Invitrogen Life Technologies) for $1 \mathrm{~h}$ at room temperature. Slides were washed, stained with DAPI, and mounted with Vectashield (Vector Laboratories). The cell count in each CB was done manually by a blinded analyzer using a Nikon Eclipse ME600 fluorescent microscope equipped with a high-resolution DXM1200C Nikon digital camera. The CB area was measured using NIH Image J software. A total of three slides per mouse, each containing three slices, were analyzed in three independent experiments. The average of apoptotic cells in the $\mathrm{CB}$ was normalized to the $\mathrm{CB}$ area of that and then normalized to the WT control average.

Statistical analysis. Statistical calculations were performed using a statistical software package (GraphPad Prism). For comparisons of two groups, means \pm SEMs were analyzed by the two-tailed unpaired Student's $t$ test. For comparisons of more than two groups, significance was determined using one-way ANOVA with Tukey's post hoc test for paired comparisons. All bars denote mean \pm SEM.

\section{Results}

Our initial studies showed that Megf10 is highly expressed in the brain, especially in the $\mathrm{CB}$ (Fig. $1 A$ ). To assess the function of Megf10 as an engulfment receptor in vivo, we generated mice with targeted deletion of the Megflo gene $\left(M e g f 10^{-/}\right)$at the KOMP. We confirmed the absence of Megf10 expression in Megf $10^{-/-}$mice by quantitative PCR and found that mice heterozygous for Megf $10\left(\mathrm{Megf10^{+/- }}\right)$ express intermediate levels of Megf10 RNA compared with WT mice (Fig. $1 B$ ).

In the developing mouse CNS, massive structural rearrangements that require a high apoptosis rate take place in the first 2 postnatal weeks (Cheng et al., 2011). Because Megf10 is highly expressed in the $\mathrm{CB}$, we hypothesized that $\mathrm{Meg} f 10^{-1-}$ mice might have a defect in the clearance of apoptotic cells and accumulate noncleared apoptotic cells in the developing CB. Using CC3 staining (Fig. 1C,D) and TUNEL (Fig. 1E), we detected increased amounts of apoptotic cells in P7 Megf10 $10^{-/-}$mice, exclusively in the CB. Interestingly, adult $M e g f 10^{-/-}$brains did not show higher numbers of apoptotic cells in the CB than WT mice (data not shown), suggesting the existence of a redundant clearance mechanism in adults. Of note, $\mathrm{Meg} f 10^{+/-}$mice have a phenotype resembling the $M e g f 10^{-/-}$mice, suggesting that efficient apopto-

\footnotetext{
(Figure legend Continued.) in the CB compared with WT littermates $\left({ }^{*} p<0.05,{ }^{*} p<0.01\right.$; WT, $n=4$ mice; Megf10 $^{+/-}, n=9$ mice; Megf10 ${ }^{-/-}, n=3$ mice). D, Representative images of anti-CC3 staining quantified in C. Scale bars: $100 \mu \mathrm{m}$; insets, $1 \mu \mathrm{m}$. $\boldsymbol{E}$, TUNEL staining of the

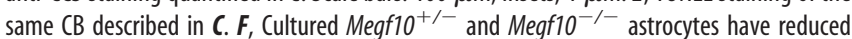
engulfment of apoptotic MEFs prelabeled with YoPRO-A488 ( $\left.{ }^{*} p<0.05,{ }^{* *} p<0.01 ; n=9\right) . \mathbf{G}$, A representative histogram of the experiment quantified in $\boldsymbol{F} . \boldsymbol{H}, \boldsymbol{I}$, Representative confocal pictures of prelabeled apoptotic CAD neurons engulfment by HEK-293T cells transfected with RFP $(\boldsymbol{H})$ or mMegf10 -RFP $(\boldsymbol{I})$. J, $\boldsymbol{K}$, Transfected HEK cells were incubated with prelabeled apoptotic CAD neurons $(\boldsymbol{J})$ or MEFs $(\boldsymbol{K})$. Scale bar, $10 \mu \mathrm{m}$. Phagocytosis is quantified by counting the percentage of cells containing an engulfed nucleus in three independent experiments done in triplicates $\left({ }^{*} p<0.05 ;{ }^{* * *} p<0.001\right)$.
}

tic cell clearance requires a certain threshold of Megf10 expression, as shown for the function of Draper and other scavenger receptors (Kay et al., 2012; Frenkel et al., 2013; Tasdemir-Yilmaz and Freeman, 2014).

Megf10 is highly enriched in astrocytes and not expressed in microglia (Cahoy et al., 2008; Hickman et al., 2013). Using flow cytometry, we found that cultured astrocytes isolated from $\mathrm{Megf} 10^{+/-}$and $\mathrm{Megf} 10^{-/-}$mice have a significant impairment in engulfment of UV-irradiated apoptotic MEFs labeled with YoPRO-A488 (Idziorek et al., 1995; Ramirez-Ortiz et al., $2013 ; \sim 60 \%$ and $\sim 65 \%$ reduction in engulfment compared with WT astrocytes, respectively; Fig. $1 F, G)$. These results indicate that Megf10 is necessary for astrocytedependent apoptotic cell clearance in the developing CB.

Because astrocytes are likely to encounter apoptotic neurons and to verify that Megf10 could indeed mediate engulfment of apoptotic neurons, we used a catecholaminergic neuronal cell line (CAD; Qi et al., 1997), irradiated with UV light, as apoptotic targets (Ramirez-Ortiz et al., 2013) and confirmed that the cells were apoptotic by staining with annexin $\mathrm{V}$ and propidium iodide (data not shown). HEK-293T-expressing mMegf10 labeled with RFP (mMegf10-RFP) engulfed $\sim 2.5$-fold more apoptotic CAD neurons and $\sim 8$-fold more apoptotic MEFs than control RFPexpressing cells (Fig. $1 H-K$ ). These data indicate that Megf10mediated engulfment is independent of the apoptotic cells type. Altogether, these experiments indicate that Megf10 is necessary and sufficient for astrocyte-dependent apoptotic neuron clearance in the developing mouse brain. Apparently, this appears to be age dependent because adult Megf $10^{-/-}$brains did not show higher numbers of apoptotic cells in the CB compared with WT mice.

Externalization of PS to the outer leaflet of the membrane by apoptotic cells is the main "eat-me" signal for engulfment by phagocytes (Fadok et al., 1992). Receptors expressed by phagocytes can bind directly to either PS or soluble bridging molecules such as C1q. To identify the Megf10 binding partner(s) on apoptotic cells, we generated a His and Flag tag secreted form of ex-hMegf10 (Fig. 2A) and quantified binding to various membrane lipids including PS in a dot blot assay. Dot blots showed strong binding of ex-hMegf10 to phosphatidic acid, moderate binding to phosphatidylinositol 4-phosphate and phosphatidylinositol $(3,4,5)$-trisphosphate, weak binding to phosphatidylinositol 4,5-bisphosphate and 3-sulfogalactosylceramide, and no binding to triglycerides, diacylglycerol, PS, phosphatidlycholine, phosphatidylglycerol, cardiopilin, phosphatidylin, cholesterol, or sphingomyelin (Fig. $2 B, C$ ).

In addition to direct recognition of PS by scavenger receptors, phagocytic cells bind to soluble bridging molecules such as $\mathrm{C1q}$ and Mfg-E8, which in turn bind PS leading to phagocytosis (Kinchen and Ravichandran, 2007). Indeed, we found that exhMegf10 binds to C1q in a dot blot assay (Fig. 2D). To confirm these results, we performed a full kinetic analysis by label-free surface plasmon resonance testing. As illustrated in the sensorgrams and summarizing table (Fig. $2 E-G$ ), dose-dependent increased interaction between Megf10 and three concentrations of C1q show that C1q binds specifically to ex-hMegf10 with a $K_{D}$ of $10^{-9} \mathrm{nM}\left(\chi^{2}<1\right)$. To determine whether the biochemical interaction observed between ex-hMegf10 and C1q translates into an interaction between cellular Megf10 and C1q, we transfected HEK-293T with full-length hMegf10 fused with GFP or with a GFP-containing vector as control. We then incubated the cells with increasing amounts of labeled $\mathrm{Clq}$ and measured cell uptake of $\mathrm{Clq}$ by the transfected cells. Flow cytometry analysis revealed 
A

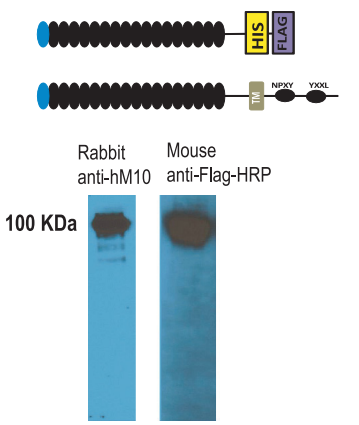

Triglyceride

Diacylglycerol

Phosphatidic Acid

Phosphatidylserine

Phosphatidylethanolamine

Phosphatidylcholine

Phosphatidylglycerol

Cardiolipin
C
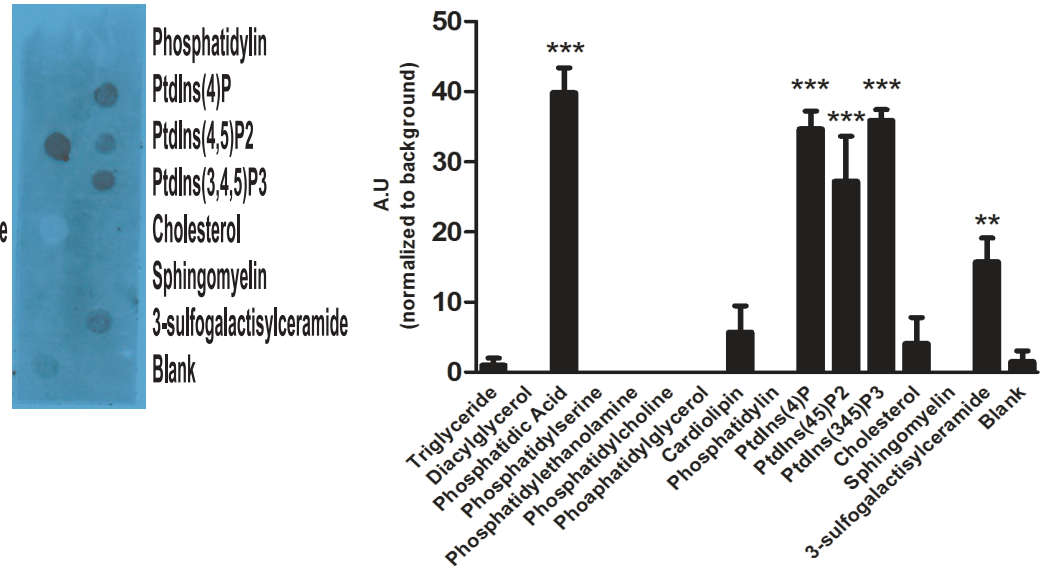
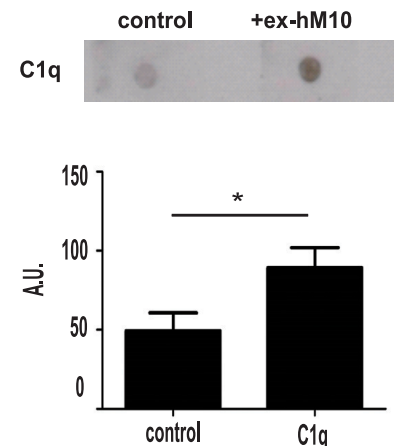

E

\begin{tabular}{|c|c|c|c|c|c|}
\hline Ligand & Analyte & KA (1/M) & KD (M) & Conc. (nM) & Chi $^{\mathbf{2}}$ \\
\hline his-Ex-hMegf10 & Phosphatidylserine & - & - & $\begin{array}{c}1000 \mathrm{nM} \\
24.6 \mathrm{uM}\end{array}$ & - \\
\hline his-Ex-hMegff10 & C1q & $1.17 \times 10^{8}$ & $8.58 \times 10^{-9}$ & $0-25$ & 0.262 \\
\hline
\end{tabular}

F

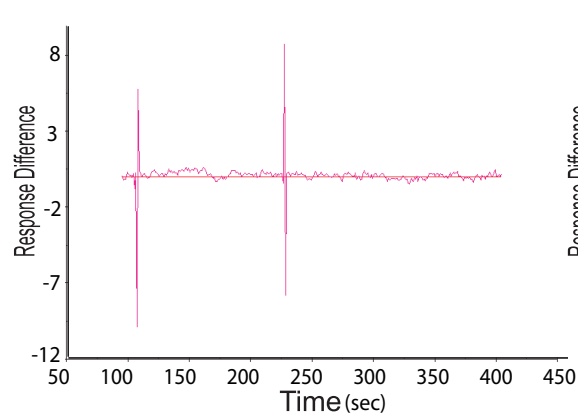

I
G

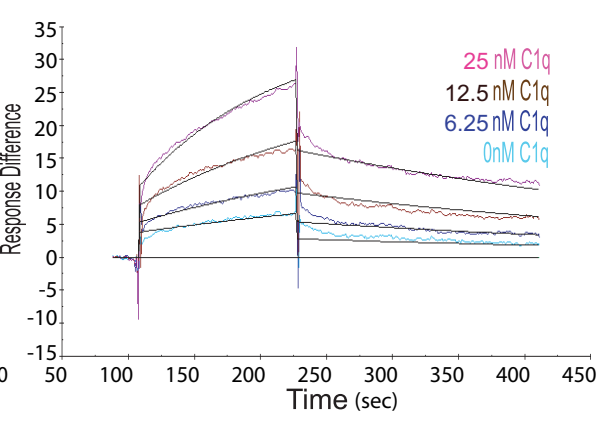

J

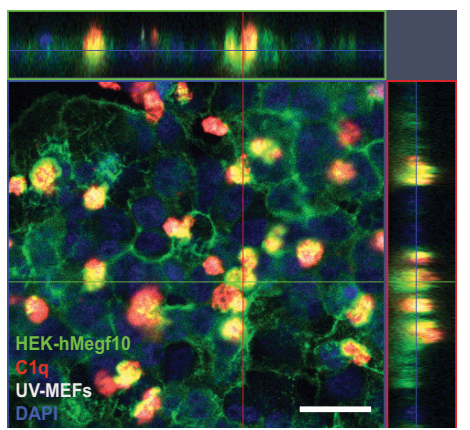

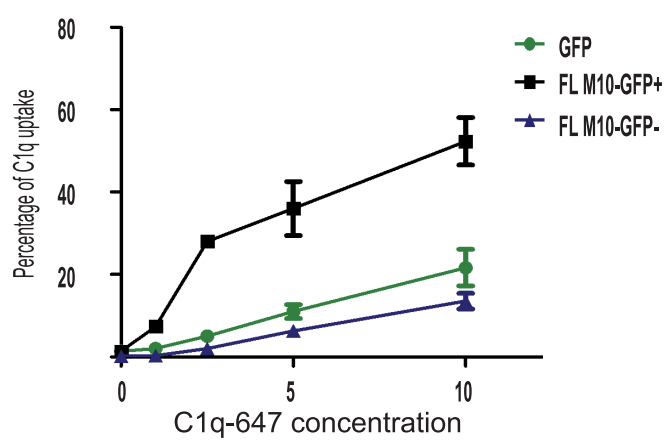

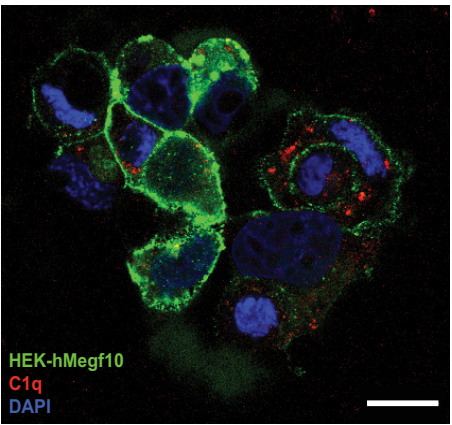

Figure 2. Megf10 does not bind directly PS but binds C $1 q$ with high affinity. $A$, Schematic depiction of secreted ex-hMegf10 tagged with His and Flag tag. Western blot confirmed that the secreted and purified protein is a single $100 \mathrm{kDa}$ band as predicted. $\boldsymbol{B}$, Dot blots showing binding of ex-hMegf10 to various membrane lipids and no binding to PS. C, Quantitative densitometry of the blot presented in $\boldsymbol{B}\left({ }^{* * *} p<0.0001 ; n=3\right.$ from 3 independent experiments). $\boldsymbol{D}$, Representative dot blot of binding of ex-hMegf10 to immobilized (1q and quantitative densitometry showing a significant increase in binding compared with secondary antibody alone $\left({ }^{*} p<0.05 ; n=4\right)$. $\boldsymbol{E}-\boldsymbol{G}$, Label-free surface plasmon resonance confirming that there is no binding to $\mathrm{PS}(\boldsymbol{F})$ and showing affinity of ex-Megf10 to $\mathrm{C} 1 \mathrm{q}$ in the nanomolar range with a highly significant $\left(\chi^{2}<1\right)$ as measured by full kinetic analysis $(\boldsymbol{G})$. $\boldsymbol{H}$, HEK expressing control GFP or full-length hMegf10 -GFP (FL M10-GFP) were incubated with the indicated amounts of Alexa-Fluor 647-C1q (C1q-647) for $1 \mathrm{~h}$ and measured by flow cytometry. FL M10 analysis was done on the GFP-positive transfected population (FL M10-GFP +) and the GFP-negative nontransfected population (FL M10-GFP - ; $n=3$ ). $I, J$, HEK cells expressing unlabeled hMegf10 were incubated with $(1 \mathrm{q}-647$ (red) (I) or (1q-647 (red) and apoptotic cells (white) (J), washed, and stained with rabbit anti-Megf10 antibody (green) and DAPI nuclear stain (blue). HEK-Megf10 cells take up (1q-647 (I) and apoptotic cells coated with C1q-647 (J). Scale bar, $20 \mu \mathrm{m}$.

that cells expressing full-length Megf10-GFP, but not GFP control, bound to Clq in a dose-dependent manner (Fig. 2H). Similar results were obtained with cells transfected with Megf10 without GFP fusion protein (data not shown and Fig. 2I). Uptake of C1q was confirmed by fluorescence microscopy. Figure $2 I$ shows a representative image of Alexa-Fluor 647-C1q (red) uptake by Megf10-transfected cells immunostained with an antiMegf10 antibody (green). To test whether C1q-labeled apoptotic 
A

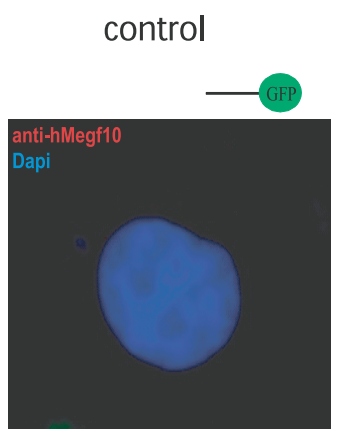

B

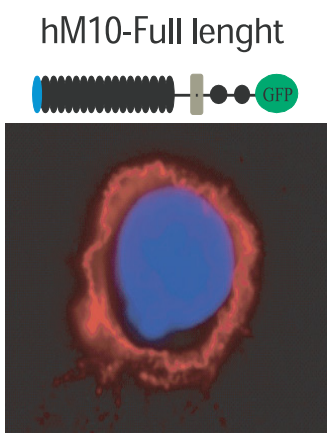

C

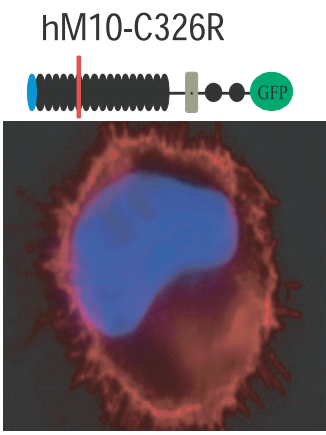

D

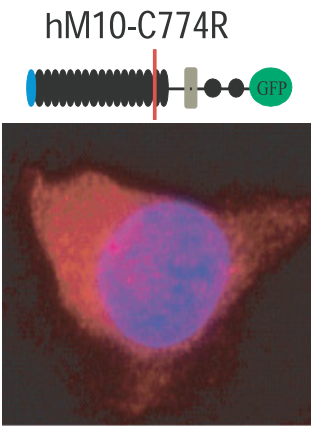

E

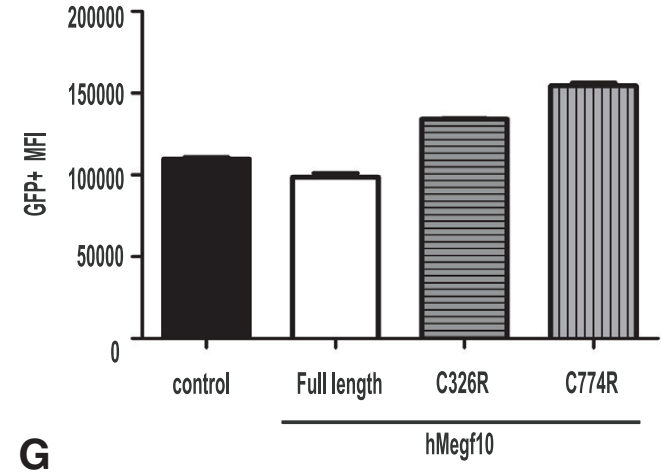

$\mathbf{F}$

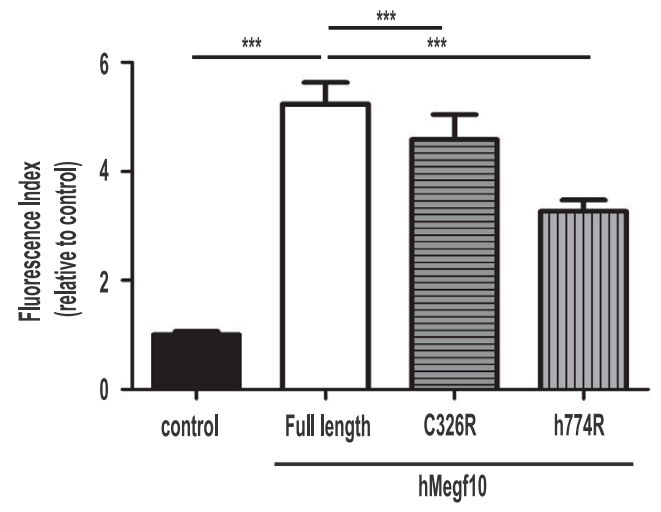

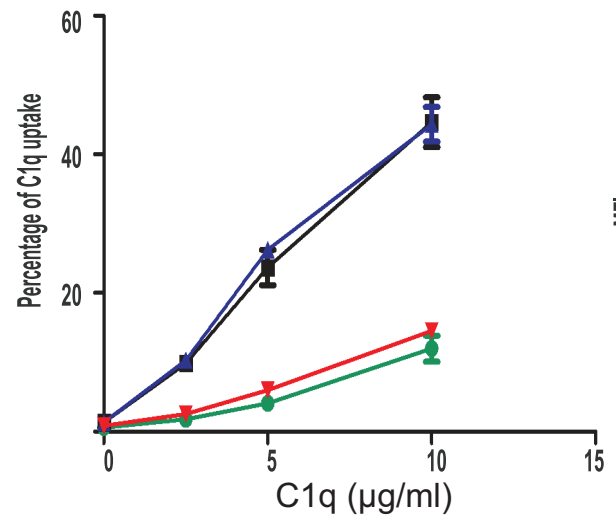

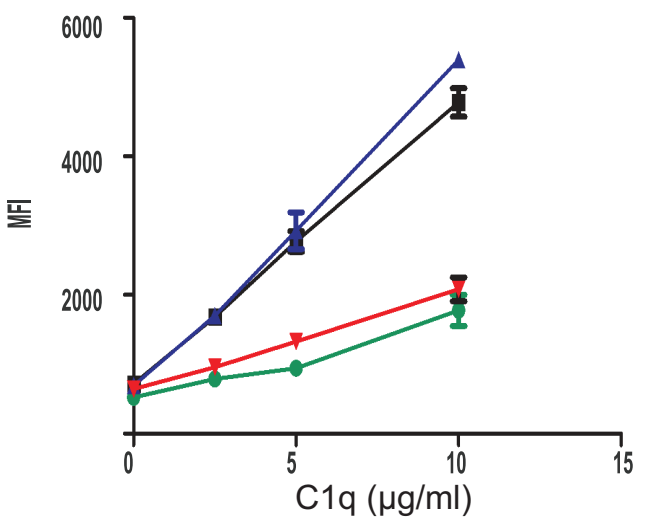

$\rightarrow$ GFP

$\rightarrow$ FL M10-GFP

\pm M10 C326R-GFP

$\rightarrow$ M10 C774R-GFP

Figure 3. EMARDD mutations impair engulfment of apoptotic cells. $\boldsymbol{A}-\boldsymbol{D}$, Anti-Megf10 staining of HEK cells expressing control $(\boldsymbol{A})$, full-length hMegf10 (B), hMegf10 -C $326 \mathrm{R}(\boldsymbol{C})$, and hMegf10 C774R (D) validating expression of Megf10 on the cell surface. $\boldsymbol{E}$, Mean fluorescent intensity (MFI) of GFP-positive cells confirm similar expression levels of Megf10 in all transfected cells. $\boldsymbol{F}$, Analysis of UV MEF uptake by HEK transfected cells shows a defect in engulfment in hMegf10 mutations (326R (22\% reduction) and C774R (53\% reduction) compared with full-length hMegf10 (*** $p<$ $0.0001 ; n=9$ from 3 independent experiment run in triplicates). $\mathbf{G}, \boldsymbol{H}$, Transfected HEK cells were incubated with the indicated amounts of Alexa-Fluor $647-C 1 q$ for $1 \mathrm{~h}$. Percentage of uptake ( $\boldsymbol{H}$ ) and $\mathrm{MFI}(\boldsymbol{I})$ was measured by flow cytometry.

cells are also taken via Megf10, we examined the uptake of apoptotic MEFs in the presence of Alexa-Fluor 647-C1q and found indeed that C1q labeled the apoptotic MEFs (white and red) and that the labeled cells are taken by HEK-Megf10 cells (Fig. 2J, green). Because C1q binds PS externalized on the surface of apoptotic cells (Kinchen and Ravichandran, 2007), these data suggest that $\mathrm{C1q}$ serves as a bridging molecule between apoptotic cells and Megf10.

Megf10 is a key regulator of myosatellite cells differentiation (Holterman et al., 2007). Genetic linkage analyses in humans indicated that recessive mutations in Megf10 result in EMARDD (Logan et al., 2011; Boyden et al., 2012). To investigate whether these specific mutations affect the function of Megf10 as an engulfment receptor, we used site-directed mutagenesis to generate GFP-labeled Megf10 expression vectors containing two different EMARDD Megf10 missense mutations: C326R and C774R. These mutations cause a substitution of cysteine with arginine, affecting the disulfide bonds at EGF-like repeats 6 and 16, respectively (Logan et al., 2011; Boyden et al., 2012). We then transfected HEK-293T with control GFP, full-length hMegf10, or Megf10 with EMARDD mutations and measured the ability of these cells to phagocytose UV-irradiated labeled apoptotic cells for $4 \mathrm{~h}$. Apoptotic cell engulfment was quantified by flow cytometry. Full-length hMegf10 or Megf10 with EMARDD mutations 
were expressed on the cell membrane and expressed in comparable levels in all transfected cells (Fig. 3A-E). As observed with mMegf10-transfected cells, engulfment levels in cells expressing full-length hMegf10 were 5.8-fold higher than control transfected cells. In contrast, cells expressing Megf10 with EMARDD mutations showed a significant defect in engulfment of apoptotic cells. Cells expressing the C774R mutation had a 53\% reduction in engulfment compared with full-length Megf10, whereas cells expressing the C326R mutation had a lower (22\%) yet statistically significant reduction in engulfment compared with full-length Megf10 (Fig. 3F). Given that Megf10 binds to C1q, we reasoned that EMARDD mutations will also affect binding to C1q. Interestingly, cells expressing EMARDD mutation C774R, which showed a more pronounced defect in apoptotic cell uptake, bound C1q as low as the GFP control cells, indicating a substantial defect in binding to C1q (Fig. $3 G, H$ ).

\section{Discussion}

Our data demonstrate that Megf10 deletion leads to defective clearance of apoptotic cells in the developing CB and strongly support a role for astrocyte Megf10 in apoptotic cell clearance in vivo. This process appears to be mediated by binding of Megf10 to the bridging molecule C1q, which binds PS expressed on the surface of apoptotic cells (Païdassi et al., 2008).

Our findings also show that clearance of apoptotic cells is evolutionarily conserved because Draper, an Megf10 ortholog expressed in Drosophila, also mediates apoptotic cell clearance (Tasdemir-Yilmaz and Freeman, 2014), indicating that this is an essential function of this family of receptors. We did not observe any accumulation of apoptotic cells in the CB of adult Megf $10^{-1-}$ animals in which baseline apoptosis is low. It is possible that clearance of apoptotic cells in the adult CNS occurs via a different pathway possibly involving another phagocytic receptors either on astrocytes or microglia, such as brain-specific angiogenesis inhibitor 1 (Park et al., 2007). It is also possible that, under conditions in which apoptosis rates are increased in the adult brain such as after injury or neurodegeneration, Megf10 may then play a similar role as observed in the developing brain. Additional research is required to investigate this possibility.

These findings also draw a parallel between the phagocytic pathways used by astrocytes for clearance of synapses and those used for the clearance of apoptotic cells. Megf10 is involved in synaptic pruning in the developing CNS (Chung et al., 2013), a process that possibly involves tagging such synapses with $\mathrm{Clq}$ (Stevens et al., 2007). Based on our findings and the published literature, we propose that synaptic pruning and engulfment of full cell corpses share similar mechanisms. Future studies are needed to further characterize the exact mechanisms that underlie each of these pathways.

Several studies showed that tyrosine phosphorylation is necessary for proper phagocytic activity of Draper (Ziegenfuss et al., 2008) and Megf10 (Scheib et al., 2012). Interestingly, a study investigating the effect of EMARDD mutations C326R and C774R on myoblast function revealed that $\mathrm{C} 774$, but not $\mathrm{C} 326$, had reduced tyrosine phosphorylation (Mitsuhashi et al., 2013). Because in our studies C774 exhibited lower ability to bind C1q, we propose that the defect in tyrosine phosphorylation seen with C774R mutation is attributable to a defect in ligand binding to this protein.

Our data also shed some light on the ligand binding domain of C1q on Megf10 and suggest that binding of C1q to Megf10 is dependent on EGF repeat 16 (where mutation C774R occurs) and not repeat 6 (where mutation C326R occurs). A detailed structure function analysis with targeted deletion of individual EGF repeats will further confirm this hypothesis.

It is not known whether EMARDD mutations affect apoptotic cell clearance or synaptic pruning in patients carrying these mutations, and it is possible that the motor dysfunction observed in EMARDD patients is not attributable to a defect in clearances of apoptotic cells but to other alterations in neuronal networks. However, our data show a potential novel effect of EMARDD mutations in the CNS. Future studies are necessary to confirm these findings in patients with these mutations.

\section{References}

Boyden SE, Mahoney LJ, Kawahara G, Myers JA, Mitsuhashi S, Estrella EA, Duncan AR, Dey F, DeChene ET, Blasko-Goehringer JM, Bönnemann CG, Darras BT, Mendell JR, Lidov HG, Nishino I, Beggs AH, Kunkel LM, Kang PB (2012) Mutations in the satellite cell gene MEGF10 cause a recessive congenital myopathy with minicores. Neurogenetics 13: 115-124. CrossRef Medline

Cahoy JD, Emery B, Kaushal A, Foo LC, Zamanian JL, Christopherson KS, Xing Y, Lubischer JL, Krieg PA, Krupenko SA, Thompson WJ, Barres BA (2008) A transcriptome database for astrocytes, neurons, and oligodendrocytes: a new resource for understanding brain development and function. J Neurosci 28:264-278. CrossRef Medline

Cheng XS, Li MS, Du J, Jiang QY, Wang L, Yan SY, Yu DM, Deng JB (2011) Neuronal apoptosis in the developing cerebellum. Anat Histol Embryol 40:21-27. CrossRef Medline

Chung WS, Clarke LE, Wang GX, Stafford BK, Sher A, Chakraborty C, Joung J, Foo LC, Thompson A, Chen C, Smith SJ, Barres BA (2013) Astrocytes mediate synapse elimination through MEGF10 and MERTK pathways. Nature 504:394-400. CrossRef Medline

Fadok VA, Voelker DR, Campbell PA, Cohen JJ, Bratton DL, Henson PM (1992) Exposure of phosphatidylserine on the surface of apoptotic lymphocytes triggers specific recognition and removal by macrophages. J Immunol 148:2207-2216. Medline

Faulkner JR, Herrmann JE, Woo MJ, Tansey KE, Doan NB, Sofroniew MV (2004) Reactive astrocytes protect tissue and preserve function after spinal cord injury. J Neurosci 24:2143-2155. CrossRef Medline

Freeman MR, Delrow J, Kim J, Johnson E, Doe CQ (2003) Unwrapping glial biology: $\mathrm{Gcm}$ target genes regulating glial development, diversification, and function. Neuron 38:567-580. CrossRef Medline

Frenkel D, Wilkinson K, Zhao L, Hickman SE, Means TK, Puckett L, Farfara D, Kingery ND, Weiner HL, El Khoury J (2013) Scaral deficiency impairs clearance of soluble amyloid-beta by mononuclear phagocytes and accelerates Alzheimer's-like disease progression. Nat Commun 4:2030. CrossRef Medline

Hickman SE, Kingery ND, Ohsumi TK, Borowsky ML, Wang LC, Means TK, El Khoury J (2013) The microglial sensome revealed by direct RNA sequencing. Nat Neurosci 16:1896-1905. CrossRef Medline

Holterman CE, Le Grand F, Kuang S, Seale P, Rudnicki MA (2007) Megf10 regulates the progression of the satellite cell myogenic program. J Cell Biol 179:911-922. CrossRef Medline

Idziorek T, Estaquier J, De Bels F, Ameisen JC (1995) YOPRO-1 permits cytofluorometric analysis of programmed cell death (apoptosis) without interfering with cell viability. J Immunol Methods 185:249-258. CrossRef Medline

Iram T, Frenkel D (2012) Targeting the role of astrocytes in the progression of Alzheimer's disease. Curr Signal Transduct Ther 7:20-27. CrossRef

Kay JN, Chu MW, Sanes JR (2012) MEGF10 and MEGF11 mediate homotypic interactions required for mosaic spacing of retinal neurons. Nature 483:465-469. CrossRef Medline

Kinchen JM, Ravichandran KS (2007) Journey to the grave: signaling events regulating removal of apoptotic cells. J Cell Sci 120:2143-2149. CrossRef Medline

Logan CV, Lucke B, Pottinger C, Abdelhamed ZA, Parry DA, Szymanska K, Diggle CP, van Riesen A, Morgan JE, Markham G, Ellis I, Manzur AY, Markham AF, Shires M, Helliwell T, Scoto M, Hübner C, Bonthron DT, Taylor GR, Sheridan E, Muntoni F, Carr IM, Schuelke M, Johnson CA (2011) Mutations in MEGF10, a regulator of satellite cell myogenesis, cause early onset myopathy, areflexia, respiratory distress and dysphagia (EMARDD). Nat Genet 43:1189-1192. CrossRef Medline

Mitsuhashi S, Mitsuhashi H, Alexander MS, Sugimoto H, Kang PB (2013) 
Cysteine mutations cause defective tyrosine phosphorylation in MEGF10 myopathy. FEBS Lett 587:2952-2957.

Païdassi H, Tacnet-Delorme P, Garlatti V, Darnault C, Ghebrehiwet B, Gaboriaud C, Arlaud GJ, Frachet P (2008) C1q binds phosphatidylserine and likely acts as a multiligand-bridging molecule in apoptotic cell recognition. J Immunol 180:2329-2338. CrossRef Medline

Park D, Tosello-Trampont AC, Elliott MR, Lu M, Haney LB, Ma Z, Klibanov AL, Mandell JW, Ravichandran KS (2007) BAI1 is an engulfment receptor for apoptotic cells upstream of the ELMO/Dock180/Rac module. Nature 450:430-434. CrossRef Medline

Prabhudas M, Bowdish D, Drickamer K, Febbraio M, Herz J, Kobzik L, Krieger M, Loike J, Means TK, Moestrup SK, Post S, Sawamura T, Silverstein S, Wang XY, El Khoury J (2014) Standardizing scavenger receptor nomenclature. J Immunol 192:1997-2006. CrossRef Medline

Qi Y, Wang JK, McMillian M, Chikaraishi DM (1997) Characterization of a CNS cell line, $\mathrm{CAD}$, in which morphological differentiation is initiated by serum deprivation. J Neurosci 17:1217-1225. Medline

Ramirez-Ortiz ZG, Pendergraft WF 3rd, Prasad A, Byrne MH, Iram T, Blanchette CJ, Luster AD, Hacohen N, El Khoury J, Means TK (2013) The scavenger receptor SCARF1 mediates the clearance of apoptotic cells and prevents autoimmunity. Nat Immunol 14:917-926. CrossRef Medline

Scheib JL, Sullivan CS, Carter BD (2012) Jedi-1 and MEGF10 signal engulf- ment of apoptotic neurons through the tyrosine kinase Syk. J Neurosci 32:13022-13031. CrossRef Medline

Stevens B, Allen NJ, Vazquez LE, Howell GR, Christopherson KS, Nouri N, Micheva KD, Mehalow AK, Huberman AD, Stafford B, Sher A, Litke AM, Lambris JD, Smith SJ, John SW, Barres BA (2007) The classical complement cascade mediates CNS synapse elimination. Cell 131:1164-1178. CrossRef Medline

Tasdemir-Yilmaz OE, Freeman MR (2014) Astrocytes engage unique molecular programs to engulf pruned neuronal debris from distinct subsets of neurons. Genes Dev 28:20-33. CrossRef Medline

Wu HH, Bellmunt E, Scheib JL, Venegas V, Burkert C, Reichardt LF, Zhou Z, Fariñas I, Carter BD (2009) Glial precursors clear sensory neuron corpses during development via Jedi-1, an engulfment receptor. Nat Neurosci 12:1534-1541. CrossRef Medline

Wyss-Coray T, Loike JD, Brionne TC, Lu E, Anankov R, Yan F, Silverstein SC, Husemann J (2003) Adult mouse astrocytes degrade amyloid-beta in vitro and in situ. Nat Med 9:453-457. CrossRef Medline

Zhou Z, Hartwieg E, Horvitz HR (2001) CED-1 is a transmembrane receptor that mediates cell corpse engulfment in C. elegans. Cell 104:43-56. CrossRef Medline

Ziegenfuss JS, Biswas R, Avery MA, Hong K, Sheehan AE, Yeung YG, Stanley ER, Freeman MR (2008) Draper-dependent glial phagocytic activity is mediated by Src and Syk family kinase signalling. Nature 453:935-939. CrossRef Medline 